\title{
Substances inertes et plantes à effet insecticide utilisées dans la lutte contre les insectes ravageurs des céréales et légumineuses au Sénégal et en Afrique de l'Ouest
}

\author{
Papa Seyni CISSOKHO ${ }^{1 *}$, Momar Talla GUEYE ${ }^{1}$, El Hadj SOW ${ }^{2}$ et Karamoko DIARRA $^{3}$ \\ ${ }^{1}$ Institut de Technologie Alimentaire, Laboratoire des Analyses Phytosanitaires, BP 2765, Dakar, Sénégal. \\ ${ }^{2}$ Université Cheikh Anta Diop, Faculté des Sciences et Technique, Département de Géologie, \\ BP 5005, Dakar, Sénégal. \\ ${ }^{3}$ Université Cheikh Anta Diop, Faculté des Sciences et Technique, Département de Biologie Animale, \\ BP 5005, Dakar, Sénégal. \\ *Auteur correspondant, E-mail : papaseynicissokho@yahoo.fr ; papseyni@gmail.com;
} Tél.: (221)702008352.

\section{RESUME}

La situation alimentaire est caractérisée au Sénégal et dans le Sahel par l'insuffisance des récoltes à laquelle s'ajoutent des pertes souvent élevées dues en grande partie aux attaques des insectes ravageurs au champ et dans les lieux de stockage. En Afrique subsaharienne, l'action des insectes déprédateurs de céréales et de légumineuses peut anéantir complètement, en quelques mois seulement, des stocks destinés aux vivres et aux semences si aucune protection n'est appliquée. Pour y apporter des solutions, les producteurs ont recours le plus souvent aux pesticides de synthèse. La résistance des insectes, les intoxications et les pollutions liées à l'utilisation des pesticides constituent de sérieux problèmes environnementaux et de santé publique. C'est ainsi que ces dernières années, de nombreux travaux ont été menés pour proposer des méthodes alternatives de protection, peu coûteuses et qui respectent l'environnement. Les insecticides naturels tels que les plantes à effet insecticide et les substances inertes (sable, cendre, terres à diatomées,...) méritent d'être valorisées afin de réduire l'utilisation des insecticides chimiques et protéger l'environnement. Ce travail basé sur une revue documentaire fouillée et actualisée vise à faire la genèse des méthodes alternatives de lutte contre les ravageurs des denrées en stockage en mettant l'accent sur les insecticides naturels et les substances inertes susceptibles d'améliorer la protection des récoltes sans danger. Les principales pratiques de stockage sont passées au peigne fin.

(C) 2015 International Formulae Group. All rights reserved.

Mots clés: Céréales, légumineuses, post-récolte, pesticides, substances inertes, plantes insecticides.

\section{INTRODUCTION}

D'après l'Organisation des Nations Unies pour l'alimentation et l'agriculture (FAO), le Fonds international de développement agricole (FIDA) et le Programme alimentaire mondial (PAM) (2013), 842 millions de personnes soit $12 \%$ de la population mondiale n'étaient pas en mesure de satisfaire leurs besoins énergétiques alimentaires entre 2011 et 2013. La grande majorité des personnes souffrant de la faim, soit 827 millions d'individus, vivent dans des pays en développement, où la prévalence de la sous-alimentation est aujourd'hui estimée à 
$14,3 \%$ de la population. L'Afrique subsaharienne n'a fait que de modestes progrès au cours de ces dernières années et reste la région à plus forte prévalence de sousalimentation. Pourtant, le Centre Technique de Coopération Agricole et Rurale (CTA) (2012) estime que le tiers des aliments produits dans les pays industrialisés est jeté ou gaspillé et que par ailleurs, la quantité de nourriture perdue, de 1,3 milliards de tonnes correspond à la production totale de l'Afrique subsaharienne.

Le déficit en céréales et légumineuses de la plupart des pays sahéliens est resté chronique ces dernières années. En 2012, les baisses de production céréalière les plus importantes ont été enregistrées en Gambie (56\%), au Tchad (49\%), au Sénégal (36\%), au Niger (31\%), en Mauritanie (34\%) et au Burkina Faso (20\%). Entre une récolte sur un temps court et une consommation annuelle, une partie non négligeable des grains et graines est perdue à la suite de diverses déprédations dont les pertes les plus importantes sont infligées par des insectes coléoptères et lépidoptères. Les estimations fournies par la FAO (2012) à une échelle plus large, indiquent qu'en Afrique subsaharienne notamment, les pertes alimentaires par habitant sont de $120-170 \mathrm{~kg} / \mathrm{an}$ et plus de $40 \%$ des pertes alimentaires sont constatées pendant la phase d'après récolte et la transformation des produits. En effet, les pertes post-récoltes de céréales, avant transformation, sont estimées à 10-20\%, soit environ 4 milliards de dollars et représentent $13,5 \%$ de la valeur totale de la production céréalière de ces pays. Selon M'ella (2011), les dommages phénotypiques causés par les insectes et les pertes qu'ils provoquent, tant au champ qu'en stockage, peuvent exposer le producteur à l'insécurité et à la précarité. Une étude récente menée par Guèye et al. (2012) a montré des dégâts et pertes causés par Sitophilus zeamais Motschulsky (Coleoptera: Curculionidae) respectivement de l'ordre de $40 \%$ et $20 \%$ en quatre mois de stockage sur du maïs non traité. Des travaux en grenier paysan ont donné la même tendance sur les dégâts et pertes (Guèye et al., 2013).

La méthode la plus répandue actuellement pour prévenir les dégâts dus aux ravageurs des grains et graines est la lutte chimique avec des insecticides, le plus souvent organophosphorés et pyréthrinoïdes. Les avantages de cette pratique sont liés à son coût qui peut être relativement faible, à sa facilité de mise en œuvre et à la durée de la protection qui peut se prolonger sur plusieurs mois. Cependant, les applications mal conduites des insecticides provoquent de sérieux inconvénients, notamment l'apparition de souches résistantes, l'intoxication chronique des consommateurs et un impact négatif sur l'environnement (Pretty et Hine, 2005).

Face à cette situation, le recours à des méthodes efficaces mais non chimiques peut atténuer entre autres les problèmes liés aux résidus présents dans les aliments. La recherche de méthodes alternatives de protection des denrées stockées par l'usage de substances inertes telles que les terres à diatomées et les substances végétales à effet insecticide est prometteuse dans la lutte contre les insectes ravageurs des denrées stockées à l'échelle du paysan. Leur utilisation pose moins de problèmes sanitaires et écologiques (Guèye, 2012). Des méthodes alternatives de protection des denrées stockées par l'usage de substances inertes et végétales à effet insecticide sont envisagées.

\section{Les insectes, principaux ravageurs des stocks de céréales et légumineuses}

Les dégâts causés sur les stocks de céréales et légumineuses par les insectes a fait l'objet de nombreux travaux en Afrique. Leur action est d'autant plus nocive que dans beaucoup de pays africains, les récoltes ne couvrent pas les besoins alimentaires et nutritionnels des populations. Caryedon serratus (olivier), Callosobruchus maculatus (F.), Acanthoscelides obtectus (Say) sont parmi les plus grands ravageurs des légumineuses en Afrique. Quant aux céréales, 
les plus connus et présentant un intérêt économique sont $S$. zeamais, Tribolium castaneum (Herbst), Sitotroga cerealella (Oliv.), Corcyra cephalonica et surtout Prostephanus truncatus (Horn). Ce dernier fut découvert au Sénégal récemment dans des stocks de maïs localisés vers le Sud-est du pays (Guèye et al., 2008a). Les pertes sur niébé peuvent atteindre $30 \%$ voire la destruction complète des stocks (Amevoin et al., 2007).

\section{Problématique de la lutte chimique contre les insectes des denrées stockées}

Pour la protection des stocks vivriers et de semences, organochlorés, carbamates, organophosphorés et pyréthrinoïdes de synthèse sont les pesticides les plus fréquemment utilisés (Guèye, 2012). D'après Isman (2006), plusieurs pays en développement ont encore recours au dichlorodiphényltrichloroéthane (DDT) et autres polluants organiques persistants (POP). En Afrique subsaharienne en particulier, environ $30 \%$ des produits commercialisés ne répondent pas aux normes de qualité internationale à cause du manque de moyen de contrôle efficace (FAO, 2001). Les insecticides posent en outre, des problèmes de disponibilité, de stockage et de coût. Selon Pesticide Action Network (PAN) Africa (2003) puis Isman (2006), les produits chimiques sont utilisés d'une façon abusive et impropre dans la plupart des pays africains. Les paysans détournent souvent des pesticides à des usages autres que ceux pour lesquels ils étaient destinés et les utilisent sans mesures de protection adéquates à cause des taux élevés d'analphabétisme. Une enquête menée au Sénégal par Guèye et al. (2008b) a établi une corrélation positive entre le niveau d'instruction des producteurs et les pratiques dangereuses quant à l'utilisation des pesticides. En effet, il est révélé l'usage de raticides, fongicides ou d'herbicides sur du maïs destiné à la consommation humaine.

$\begin{array}{lrrr}\text { Thiam et } & \text { Ducommun (1993) } \\ \text { évoquaient une contamination } & \text { de }\end{array}$

l'environnement par les pesticides à toutes les périodes - production - mélange - applications des produits - transport et lors des semences des grains de céréales et légumineuses. Les pesticides peuvent être transportés soit par le vent au cours du traitement, soit par les grains traités après les semis dans les champs, soit par le biais du système d'égouts et par les ruissellements en provenance des champs et des lieux de traitement pour se retrouver dans les étendues d'eau où ils auront la possibilité de pénétrer dans la chaîne alimentaire.

Les travailleurs, les animaux domestiques et tous les autres êtres vivants dans les zones agricoles sont ainsi exposés aux contaminations soit par l'air qu'ils respirent, soit par l'eau qu'ils boivent ou la nourriture qu'ils consomment. Dans les champs agricoles, les oiseaux pollinisateurs et granivores peuvent absorber les pesticides par leurs pattes ou par leur peau, les inhaler ou les ingérer avec les grains ou avec les insectes contaminés. L'utilisation des pesticides même à très faibles doses est souvent associée à des problèmes de santé et d'environnement. Les pesticides sont, de par leur nature, des produits dangereux et toxiques même à très faibles doses (Carlos, 2006). Selon Isman (2006) un nombre important de travailleurs dans les pays tropicaux et subtropicaux sont intoxiqués ou tués chaque année par des pesticides toxiques à effets aigus dont ils ignorent le mode d'emploi. L'utilisation inconsidérée des pesticides chimiques a d'autres conséquences néfastes, notamment la réduction de la biodiversité, la destruction d'une grande partie des organismes utiles tels que les organismes décomposeurs qui participent à la construction de l'humus et aux cycles biogéochimiques ainsi que de nombreux prédateurs des parasites (Glitho et al., 2008).

De nombreux pays, notamment ceux de l'Europe et de l'Amérique comme le Canada tout comme le Comité inter Etats de lutte contre la sécheresse au Sahel (CILSS) ont instauré une réglementation stricte vis-à-vis des pesticides afin de limiter leur utilisation 
entraînant le retrait de nombreux polluants organiques persistants (POPs) du secteur de la production alimentaire. Toutefois, cette réglementation n'est pas souvent appliquée en Afrique. C'est pourquoi, pour Glitho et al. (2008) les moyens naturels de contrôle constituent des éléments importants des systèmes de production. Les insecticides d'origine minérale et végétale sont parmi les sources les plus prometteuses pour les pays en développement.

\section{Méthodes alternatives à la lutte chimique dans la protection des stocks Substances inertes}

L'utilisation des poudres inertes naturelles joue un rôle important dans la conservation des denrées stockées. Leur principal avantage repose sur leur innocuité (Golob, 1997). Selon Banks et Fields (1995), Il existe quatre types de poudres inertes desséchantes abrasives, en l'occurrence la terre (Argile poudrée, sable, terre...), la terre à diatomées (Protect-It, Dryacide, Silicosec...), les gels siliceux, et les poudres inertes non siliceuses [(roches phosphatées, terre soufrée, chaux (hydroxyde de calcium), calcaire (carbonate de calcium), sel (chlorure de sodium)].

D'après Ebeling (1971), l'insecte meurt lorsqu'il a perdu $60 \%$ de son eau ou $30 \%$ de sa masse corporelle. La cendre et le sable fin sont utilisés pour traiter les stocks selon des proportions et des pratiques qui varient suivant les régions. Ces matériaux pulvérulents remplissent les vides entre les graines et constituent une barrière à la progression des femelles cherchant à pondre. Ces matériaux auraient un rôle abrasif sur les insectes et entraîneraient leur déshydratation en adsorbant ou abrasant le film lipoïdique protégeant leur cuticule (Cruz et Troude, 1988). Les doses préconisées varient de 1-2\% du poids des denrées à $0,25 \%-0,5 \%$ suivant la qualité des poudres et la teneur en eau du grain qui ne doit pas dépasser $15 \%$. L'utilisation de la cendre et du sable est limitée au stockage traditionnel en greniers.
L'efficacité de ce moyen réside dans le fait qu'il entrave la mobilité des insectes. Au Mexique, les cendres volcaniques du mont Chichonal appliquées au maïs à $1 \%$ ont montré un bon contrôle de $P$. truncatus (Sanchez et al., 1989). Les zéolites, autres substances minérales inertes possédant des propriétés physiques semblables à celles des terres à diatomées ont donné des résultats satisfaisants à des doses de 0,$5 ; 0,75$ et $1 \mathrm{~g} / \mathrm{kg}$ de blé au bout de trois semaines avec $S$. oryzae (L.) (96-98\%), Rhyzopertha dominica (F.) (70-82\%) et T. castaneum (100\%) (Kljajic et al., 2010).

\section{Cas des terres à diatomées}

Prises comme méthode environnementale acceptable, les terres à diatomées ont été les plus étudiées dans le domaine de l'utilisation de substances inertes dans la protection des stocks. Les terres à diatomées sont des dépôts géologiques de diatomites, qui sont des couches sédimentaires fossilisées d'algues microscopiques appelées diatomées. Le constituant majeur de la diatomite est de la silice $\left(\mathrm{SiO}_{2}\right)$ avec en plus des quantités variables d'autres minéraux dont l'Aluminium, l'Oxyde de fer, l'Hydroxyde de calcium, le Magnésium et le Sodium. L'efficacité insecticide de la terre à diatomées peut varier dans des limites assez larges selon l'origine du dépôt fossile exploité car tous les gisements n'ont pas la même composition (Korunic, 1998). Les facteurs importants pour l'efficacité d'une terre à diatomées sont une concentration en silice supérieure à $80 \%$, un pourcentage élevé de fines particules, un $\mathrm{pH}$ inférieur à 8,5 et une densité inférieure à 300 g/l (Korunic, 1998).

Les terres à diatomées ont plusieurs avantages sur les denrées stockées à savoir une faible toxicité mammaire; en effet, elles peuvent assurer une protection à long terme contre les insectes ravageurs et peuvent facilement être enlevées de la graine durant le traitement (Athanassiou et al., 2005). Ces derniers ont mis en exergue l'efficacité et la persistance de trois formulations 
commerciales de terres à diatomées à savoir Insecto, PyriSec, et SilicoSec contre $S$. oryzae sur le blé et l'orge avec une forte mortalité des charançons $(92 \%)$ et une réduction des émergences après neuf mois. Saez et Fuentes (2007) ont montré quant à eux l'importance de l'origine (eau douce ou marine) et du mode d'application des terres à diatomées sur Cryptolestes ferrugineus, $R$. dominica, $S$. oryzae et $S$. granarius par une différence d'adhérence des particules. Plusieurs facteurs sont connus pour affecter l'activité insecticide des terres à diatomées: l'humidité relative, la température, la source géologique, le type de grains, les espèces d'insectes, leur stade de développement et leur densité (Fields et Korunic, 2000a, Arthur, 2002). Ces facteurs sont à l'origine des difficultés à obtenir des résultats reproductibles au laboratoire ou entre les laboratoires (Fields et al., 2000b). Vayias et al. (2006) ont constaté que la terre à diatomée est beaucoup plus efficace lorsqu'elle est appliquée sur du blé plutôt que sur du maïs; de larges espaces entre les grains de maïs pourraient permettre aux insectes de ramper à travers et éviter les zones où la concentration de la terre à diatomées est élevée. Au laboratoire, Eberling (1971) a constaté que la perte ou la baisse de l'efficacité des poussières inertes peut être attribuée au ralentissement de la capacité d'adsorption par contact direct des couches lipidiques dans des conditions humides. Les résultats de Khakame et al. (2012) ayant obtenu de faibles mortalités de $S$. zeamais sur les grains de maïs ayant une humidité de $16 \%$ par rapport à ceux ayant des humidités respectives de 10,12 et $14 \%$ confortent ce dernier auteur.

Substances végétales dans la gestion des insectes ravageurs des denrées stockées

Des programmes de recherche sur les plantes à effet insecticide ont débouché sur de très nombreuses communications et publications scientifiques à travers l'Afrique subsaharienne (Seck et al., 1996; Guèye, 2012, Diouf et al., 2014). Ces travaux ont largement contribué à une meilleure compréhension des activités insecticide, répulsive et antiappétante de certaines substances végétales vis-à-vis des insectes.

\section{Plantes à effet insecticide}

Plusieurs plantes testées sur les Coléoptères, ravageurs du maïs, du manioc, du niébé et du haricot ont prouvé leur potentiel insecticide (Glitho et al., 2008). Les Méliacées sont parmi les plantes les plus expérimentées pour leur effet de contact et le neem en est sans doute l'espèce la plus étudiée dans ce cadre (Facknath, 2006). En effet, il a été démontré que l'extrait du neem constitue un mélange de plus de 100 composés qui sont responsables de la mortalité des insectes (Addea-Mensah, 1998). La poudre des grains de neem à un taux de $2 \mathrm{~g} / \mathrm{kg}$ de maïs a causé $100 \%$ de mortalité de $S$. oryzae après dix jours de traitement (Mahanti, 2002). Srinivasan (2008), après neuf mois de traitement de pois à l'huile des grains de neem à 10 et $5 \mathrm{ml} / \mathrm{kg}$ a rapporté que les dégâts étaient respectivement de l'ordre de 9,2 à $15 \%$ en comparaison au témoin non traité où ils étaient de 96\%. D'autres plantes d'autres genres ont aussi été appliquées avec succès contre les ravageurs des denrées stockées. Au Cameroun, de la cendre de feuilles de Eucalyptus grandis sur $S$. zeamais à une dose de $20 \mathrm{~g} / 2 \mathrm{~kg}$ a montré une bonne efficacité sur du maïs, au bout de six mois (Akob et Ewete, 2007). Guèye et al. (2012) ont quant à eux démontré l'efficacité par contact de poudre d'épis vides de maïs avec une efficacité équivalente à celle du pyrimyphos méthyl à une dose égale à $4 \%$ dans le contrôle de $S$. zeamais. Par ailleurs, il a été mis en évidence que quand les doses augmentent, l'application de la poudre d'épis allonge le développement larvaire et diminue à la fois l'intervalle de temps dans lequel les adultes émergent de 35 à $96 \%$ et la fécondité de 89 à $99 \%$.

\section{Huiles essentielles}

Plusieurs travaux ont montré que les huiles essentielles des plantes sont biologiquement actives contre les ravageurs 
des denrées stockées par contact direct ou par inhalation. Le spectre d'action des huiles essentielles est très diversifié; elles jouent un rôle de régulateur de croissance et possèdent des propriétés antimicrobiennes et antioxydantes (Goudoum, 2010). Les huiles essentielles de $O$. gratissimum et Xylopia aethiopica ont montré une activité insecticide sur S. zeamais (Ngassoum et al., 2003). Toutefois, la sensibilité des larves de $T$. castaneum à ces deux huiles essentielles diminue avec l'âge (Kouninki et al., 2005). Rajendran et Spiranjini (2008) ont montré que les huiles essentielles appartenant principalement aux Apiaceae, Lamiaceae, Myrtaceae et leurs composants dont les monoterpénoides testées pour leur toxicité fumigène sont efficaces contre les ravageurs des denrées stockées y compris $S$. zeamais et T. casteneum. Nguemtchouin et al. (2010) ont obtenu $100 \%$ de mortalité à la dose de $10 \%$ $(\mathrm{p} / \mathrm{p})$ après utilisation de l'huile essentielle récoltée à partir du mélange de kaolinite et d'huile essentielle de $X$. aethiopica. Des tests en grenier ont permis de garder des épis de maïs en sandwich avec Hyptis spicigera pendant deux années successives sans aucune infestation malgré que les greniers soient infestés artificiellement par sept couples de $S$. zeamais et autant de $T$. castaneum (Guèye, 2012). Ce dernier a montré que les effluves issues de la plante essentiellement composés de monoterpènes dont $\alpha$-pinène $(38.9 \%), \beta$ pinène $(14.7 \%)$ et caryophyllène (12.6\%) ont éliminé les adultes introduits et empêché toute possibilité de réinfestation au cours du stockage.

Quelques expériences sénégalaises en matière de lutte par des plantes à effet insecticide

La bruche du niébé $C$. maculatus a été l'une des espèces qui très tôt a attiré le plus d'attention dans les programmes de lutte contre les ravageurs. Seck (1994) a testé près d'une dizaine de plantes entre autres contre les bruches du niébé et de l'arachide. Parmi celles-ci, B. senegalensis a fait l'objet de recherches très poussées. L'extrait acétonique de fruits de $B$. senegalensis montre un puissant effet fumigant sur $C$. maculatus et $S$. cerealella. Le dosage quantitatif des composés actifs de B. senegalensis et les valeurs de $\mathrm{CL}_{50}$ obtenues des tissus de cette plante comparées à celles des molécules pures montrent que l'activité biologique de $B$. senegalensis est due à la libération de MITC à partir d'un glucosinolate précurseur, la glucocapparine contenue dans les feuilles et fruits (Seck et al., 1993). Dans ce cadre, des feuilles fraîches broyées de B. senegalensis appliquées sur graines de niébé infestées par C. maculatus, à la concentration de 2 à $4 \%$ $(\mathrm{P} / \mathrm{P})$ ont montré une activité biologique se traduisant par une mortalité de 63 à 100\% chez les adultes dans un délai de 24 à 48 heures, avec une inhibition de l'émergence et des dégâts de la population $\mathrm{F} 1$ de $C$. maculatus (Seck et al., 1993). Sur arachide, Guèye et al. (2011) ont obtenu avec les fruits et feuilles frais de cette plante une mortalité totale de $C$. serratus respectivement aux doses de 3 et $6 \%$. Le stade physiologique de l'organe s'est montré très important dans l'efficacité de la plante sur les insectes car celle-ci donne un meilleur rendement à l'état vert (Guèye, 2012). Par la suite, des dosages de la glucocapparine ont montré le rôle de la période et de la localité de récolte sur l'efficacité des organes de $B$. senegalensis (Guèye et al., 2013a). Le dosage du principe actif, le MITC sur des grains soumis à une fumigation a révélé que ce dernier restait dans les grains et se présente donc comme un résidu au même titre que les pesticides (Guèye et al., 2013b)

Divers autres travaux surtout de laboratoire ont été menés pour expérimenter des solutions contre les bruches de l'arachide et autres déprédateurs des céréales et légumineuses. Des extraits à l'éther de pétrole et au méthanol obtenus de poudre de feuilles de Lantana camara et d'Azadirachta indica ont montré une même efficacité sur les œufs de $C$. serratus avec une mortalité de $90 \%$ à la concentration de $0,1 \mathrm{gm} / 1$ (Guèye, 2008). De même, Thiaw et Sembène (2010) ont abouti à 
des essais concluants contre la bruche de l'arachide avec des extraits de Calosopris procera. Sur base d'application d'extraits au chloroforme, méthane et cyclohexane de Crataeva religiosa, Diouf et al. (2014) ont révélé une plus forte sensibilité de $C$. maculatus que $S$. zeamais avec dans les deux cas une mortalité plus élevée avec les extraits au chloroforme.

Spinosad, une nouvelle opportunité dans la protection des denrées stockées

Le spinosad est perçu comme un biopesticide commercial à risque réduit utilisé dans la gestion de nombreuses espèces d'insectes ravageurs (Hertlein et al., 2011). Son activité est attribuée aux spinosynes A et D qui sont des produits de fermentation d'une bactérie actinomycète du sol, Saccharopolyspora spinosa. Selon ces mêmes auteurs, le spinosad serait efficace contre les insectes des denrées les plus communs ayant surtout un impact économique avéré : $S$. zeamais, $P$. truncatus, T. castaneum, $R$. dominica, Cryptolestes pusillus, Plodia interpunctella, Corcyra cephalonica et Sitotroga cerealella. Chintzoglou et al. (2008) ont rapporté que la mortalité de $S$. oryzae exposé pendant quatorze jours sur le blé traité avec spinosad aux doses de $0,0625,0,1875$ et 0,625 ppm s'échelonne entre 83 et $100 \%$.

Cependant, une étude antérieure de Vayias et al. (2006) avait démontré que quoique les performances du spinosad ne soient pas affectées par le type de grain, son efficacité sur le maïs est moins stable au-delà de 6 mois. Ces derniers le présentent comme une solution sûre du fait de sa faible toxicité mammaire $\left(\mathrm{DL}_{50}\right.$ oral sur rat $>5000 \mathrm{mg} / \mathrm{kg}$ de poids corporel).

\section{Conclusion}

L'un des facteurs clés de la sécurité alimentaire demeure une bonne conservation des récoltes. Par conséquent, une stratégie phytosanitaire à même de contenir les déprédations à des niveaux inférieurs au seuil de nuisibilité économique est nécessaire. Les plantes à effets insecticides et les substances inertes ainsi que les entomopathogènes sont présentés de nos jours comme une alternative aux pesticides de synthèse. Bien qu'étant moins rémanents que les insecticides de synthèse, ils peuvent présenter moins de risques d'accoutumance pour les insectes ainsi que la présence de résidus dans les aliments. Il serait donc intéressant d'approfondir les investigations sur leur potentiel biocide pour asseoir une lutte raisonnée et efficace contre les ravageurs des denrées alimentaires stockées.

\section{REFERENCES}

Addea-Mensah I. 1998. The uses of the neem (Azadirachta indica) in Ghana and their relations of the chemical constituents and Biological activities. Proc. Seminar held in Dodowa: the potentials of the neem trees in Ghana, 11-26.

Akob CA, Ewete FK. 2007. The efficacy of ashes of four locally used plant materials against Sitophilus zeamais (Coleoptera: Curculionidae) in Cameroon. Int. J. Trop. Insect Sci., 27(1): 21-26.

Amevoin K, Sanon A, Apossaba M, Glitho IA. 2007. Biological control of bruchids infesting cowpea by the introduction of Dinarmus basalis (Rondani) (Hymenoptera: Pteromalidae) adults into farmers stores in West Africa. J. Stor. Prod. Res., 43: 240-247.

Arthur FH. 2002. Survival of Sitophilus oryzae (L.) on wheat treated with diatomaceous earth: impact of biological and environmental parameters on product efficacy. J. Stor. Prod. Res., 38(3): 305-313.

Athanassiou CG, Kavallieratos NG, Economou LP, Dimizas CB, Vayias BJ, Tomanovic S, Milutinovic M. 2005. Persistence and efficacy of three diatomaceous earth formulations against Sitophilus oryzae (Coleoptera: Curculionidae) on wheat and barley. $J$. Econ. Entomol., 98: 1404-1412.

Banks JH, Fields P. 1995. Physical methods for insect control in stored-grain 
ecosystems. in Stored- Grain Ecosystems, Jayas DS, White, Muir WE (Eds). Marcel Dekker Inc: New York, 353-409.

Carlos JSP. 2006. «Exposition humaine aux pesticides: un facteur de risque pour le suicide au Brésil». Vertigo-La revue en Science de l'Environnement, 7: 18.

Chintzoglou GJ, Athanassiou CG, Markoglou AN, Kavallier NG. 2008. Influence of commodity on the effect of spinosad dust against Rhyzopertha dominica (F.) (Coleoptera: Bostrychidae) and Sitophilus oryzae (L.) (Coleoptera : Curculionidae). Int. Pest. Mngt. J., 54(4): 277-285.

Cruz JF, Troude F. 1988. Conservation des Grains en Régions Chaudes. Collection du Ministère de la Coopération et du Développement. Techniques Rurales en Afrique. CEEMAT / CIRAD: Montpellier ; 548.

CTA. 2012. Gaspillage: occasions manquées dans le combat pour accroître la sécurité alimentaire. Note politique $\mathrm{N}^{\circ} 7,4 \mathrm{p}$.

Diouf EHG, Samb A, Seck D, Diop M. 2014. Phytochemical and insecticidal study of three organic extracts of Crataeva religiosa Forst on Sitophilus zeamais and Callosobruchus maculatus. Int. Res. J. Phram. App. Sci., 4(4) 13-18.

Ebeling W. 1971. Sorptive dusts for pest control. An. Review of Entomol., 16: $123-158$

Facknath S. 2006. Combination of neem and physical disturbance for the control of four insect pests of stored products. Int. J. Tropical Ins. Sci., 26(1) 16-27.

FAO (Organisation des Nations Unies pour l'alimentation et l'agriculture), FIDA (Fonds international de développement agricole), PAM (Programme alimentaire mondial). 2013. L'état de l'insécurité alimentaire dans le monde 2013. Les multiples dimensions de la sécurité alimentaire. Rome. FAO. Septembre 2013. 63 p.
FAO (Organisation des Nations Unies pour l'alimentation et l'agriculture). 2001. Les pesticides périmés menacent l'Afrique d'une catastrophe sanitaire. Afrique relance. ONU, vol.15. $42 \mathrm{p}$.

FAO (Organisation des Nations Unies pour l'alimentation et l'agriculture). 2012. Gaspillages dans le monde, ampleur, causes et prévention. Division des infrastructures rurales et des agro industries, 44p.

Fields PG, Korunic Z. 2000a. The effect of grain moisture content and temperature on the efficacy of diatomaceous earths from different geographical locations against stored-product beetles. J. Stored Prod. Res., 36: 1-13.

Fields PG, Korunic Z. 2000b. Diatomaceous earth to control Cryptolestes ferrugineus (Coleoptera: Cucujidae) in stored barley in farm granaries. Canadian Entomol., 132: 247-258.

Glitho IA, Ketoh KG, Nuto PY, Amevoin SK, Huignard I. 2008. Approches non toxiques et non polluantes pour le contrôle des populations d'insectes nuisibles en Afrique du Centre et de l'Ouest; 207-217. In Biopesticides d'Origine Végétale (2 ${ }^{\text {ème } e ́ d n), ~ R e g n a u l t-~}$ Roger $\mathrm{C}$, Philogène BJR, Vincent $\mathrm{C}$ (eds). Lavoisier, TEC \& DOC: Paris ; $550 \mathrm{p}$.

Golob P. 1997. Current status and future perspectives for inert dusts for control of stored product insects. J. Stored Prod. Res., 33: 69-79.

Goudoum A. 2010. Impact des huiles essentielles sur le potentiel technologique et nutritionnel des grains et farine de maïs au cours du stockage. Thèse de Doctorat, ENSAI, Université de Ngaoundéré, Cameroun, 180 p.

Guèye MT, Goergen G, Badiane D, Hell K, Lamboni L. 2008a. First report on occurrence of the larger grain borer Prostephanus truncatus (Horn) (Coleoptera: Bostrichidae) in Senegal. $J$. of African Entomol., 16(2): 309-311. 
Guèye MT, Badiane M, Ndiaye AB, Mbaye I, Diouf M, Ndiaye S. 2008b. La protection des stocks de maïs au Sénégal: enquêtes sur les pratiques d'utilisation des pesticides et plantes à effet insecticide en milieu paysan. ITA Echos, $\mathrm{N}^{\circ} 3,12$.

Guèye MT, Seck D, Wathelet JP, Lognay G. 2011. Lutte contre les ravageurs des stocks de céréales et de légumineuses au Sénégal et en Afrique occidentale. Biotechnol. Agron. Soc. Environ., 15(1): 187-198.

Guèye MT. 2012. Gestion intégrée des ravageurs de céréales et de légumineuses stockées au Sénégal par l'utilisation de substances issues de plantes. Thèse de doctorat, Université de Liège Gembloux Agro-Bio Tech, 216 p.

Guèye MT, Cissokho PS, Goergen G, Ndiaye S, Seck D, Guèye G, Wathelet JP, Lognay G. 2012. Efficacy of powdered maize cobs against the maize weevil Sitophilus zeamais (Coleoptera: Curculionidae) in stored maize in Senegal. Int. J. Trop. Insect Sci., 32: 1-7.

Guèye MT, Goergen G, Ndiaye S, Asiedu EA, Lognay G, Seck D. 2013a. Efficiency of traditional maize storage and control methods in rural grain granaries: a case study from Senegal. Tropicultura, 31(2): 39-46.

Gueye MT, Seck D, Diallo A, Trisman D, Fischer C, Barthelemy JP, Wathelet JP, Lognay G. 2013b. Development of a Performant Method for Glucocapparin Determination in Boscia senegalensis Lam Ex. Poir.: A Study of the Variability. Am. J. Anal. Chem., 4: 104110.

Hertlein MB, Thompson GD, Subramanyam B, Athanassiou CG. 2011. Spinosad: A new natural product for stored grain protection. J. Stored Prod. Res., 47(3): 131-146.

Isman MB. 2006. Botanica1 insecticides, deterrents, and repellents in modem agriculture and an increasing1y regulated world. Ann. Rev. Entomol., 51: 45-66.

Khakame SK, Likhayo P, Olubayo FM, Nderitu JH. 2012. Effect of grain moisture content and storage time on efficacy of inert and botanical dusts for the control of Sitophilus zeamais in stored maize. J. Stored Prod. Postharvest Res., 3(10): 145-151.

Kljajić P, Andrić G, Adamović M, Pražić Golić M. 2010. Laboratory evaluation of insecticidal effectiveness of a natural zeolite formulation against Sitophilus oryzae (L.), Rhyzopertha dominica (F.) and Tribolium castaneum (Herbst) in treated wheat. 10th Int. Working Conf. on Stored Prod. Protection, 863-868.

Korunic Z. 1998. Diatomaceous earths, a group of natural insecticides. J. Stored Prod. Res., 34: 87-97.

Kouninki H, Haubruge E, Noudjou FE, Lognay G, Malaisse F, Ngassoum MB, Goudoum A, Mapongmetsem PM, Ngamo LS, Hance T. 2005. Potential use of essential oils from Cameroon applied as fumigant or contact insecticides against Sitophilus zeamais Motsch. (Coleoptera: Curculionidae). Commun. Agric. Appl. Biol. Sci., 70(4): 787-792.

Mahanti V. 2002. Ecology and management of stored grain pests of maize. $\mathrm{PhD}$ Thesis, Acharya N. G. Ranga Agric. Univ., Hyderabad, Andhra Pradesh (India).

M'ella JGM. 2011. Préface. In Insectes Ravageurs des Graines de Légumineuses: Biologie des Bruchinae et Lutte Raisonnée en Afrique. Huignard J, Glitho IA, Monge J-P, Regnault-Roger C (eds). Collection Update Sciences \& Technologies. Eds Quae: France; 145.

Ngassoum MB, Ngamo TLS, Maponmetsem PM, Jirovertz L, Buchbauer G. 2003. Investigation of medicinal arometic plants from Cameroon: GC/FID, GC/MS and olfactoric analyses of essential oils Ocimum suave Willd. (Lamiaceae). Acta Pharmaceutica Turcica, 45: 69-75. 
Nguemtchouin MMG, Ngassoum MB, Ngamo L, Cretin M, Gaudu X. 2010. Insecticidal activities of powdered formulation base on essential oil of Xylopia aethiopica and kaolinite clay against Sitophilus zeamais. J. of crop Protection, 29: 985999.

PAN Africa. 2003. Les pesticides au Sénégal ( $2^{\text {ème }}$ édn). PAN Africa ; 56p.

Pretty J, Hine R. 2005. Pesticide use and the environment in The pesticide detox Towards a More Sustainable Agriculture. EARTHSCAN: London, Sterling, VA; 293 p.

Rajendran S, Sriranjini V. 2008. Plant products as fumigants for stored-product insect control. J. Stored Prod. Res., 44: 126-135.

Saez A, Fuentes MVH. 2007. Comparison of the desiccation effects of marine and freshwater diatomaceous earths on insects. J. Stored Prod. Res., 43: 404409.

Sanchez-Arroyo H, Lagunes-Tejeda A, Llanderal-Cazares C. 1989. Satividad de polvos minerales para el combate de Prostephanus truncatus (Horn) y Sitophilus zeamais Motschulsky, en maiz almacendo. Agrociencia, 76: 4758.

Seck D, Lognay G, Haubruge E, wathelet JP, Marlier M, Gaspar C, Severin M. 1993. Biological activity of the shurd Boscia senegalensis (Pers.) Lam. Ex Poir. J. Chem. Ecol., 19(2): 377-389.

Seck D. 1994. Développement de méthodes alternatives de contrôle des principaux insectes ravageurs des denrées emmagasinées au Sénégal par l'utilisation de plantes indigènes. Thèse de Doctorat, Faculté des Sciences Agronomiques de Gembloux. 192 p.

Seck D, Lognay G, Haubruge E, Marlier M, Gaspar C. 1996. «Alternative Protection of Cowpea Seeds against Callosobruchus maculatus F. (Coleoptera: Bruchidae) using Hermetic Storage alone or in Combination with Boscia senegalensis Pers. Lam. Ex. (Capparaceae) on stored grain insects. $J$. Stored Prod. Res., 32: 39-44.

Srinivasan G. 2008. Efficacy of certain plant oils as seed protectant against pulse beetle, Callosobruchus chinensis Linn, on pigeon pea. Pest. Res. J., 20(1): 1315.

Thiam B, Ducommun G. 1993. Protection naturelle des végétaux en Afrique. ENDA, Tiers monde, Dakar. 213p.

Thiaw C, Sembène M. 2010. Bioactivity of crude extracts and fractions extract of Calotropis procera AIT. on Caryedon serratus (OL.) insect pest of peanut stocks in Senegal. Int. J. Biol. Chem. Sci., 4(6): 2220-2236.

Vayias BJ, Athanassiou CG, Kavallieratos NG, Tsesmeli CD, Buchelos CT. 2006. Persistence and efficacy of two diatomaceous earth formulations and a mixture of diatomaceous earth with natural pyrethrum against Tribolium confusum Jacquelin du Val (Coleoptera: Tenebrionidae) on wheat and maize. Pest Man. Sci., 62: 456-464. 